\title{
Identified particle production and freeze-out properties in heavy-ion collisions at RHIC Beam Energy Scan program
}

\author{
Sabita Das (for the STAR collaboration) $)^{1, a}$ \\ ${ }^{1}$ Institute of Physics, Bhubaneswar-751005, India
}

\begin{abstract}
The first phase of Beam Energy Scan (BES) program at the Relativistic Heavy-Ion Collider (RHIC) was started in the year 2010 with the aim to study the several aspects of the quantum chromodynamics (QCD) phase diagram. The Solenoidal Tracker At RHIC (STAR) detector has taken data at $\sqrt{s_{N N}}=7.7,11.5,19.6,27$, and $39 \mathrm{GeV}$ in $\mathrm{Au}+\mathrm{Au}$ collisions in the years 2010 and 2011 as part of the BES programme. For these beam energies, we present the results on the particle yields, average transverse mass and particle ratios for identified particles in mid-rapidity $(|y|<0.1)$. The measured particle ratios have been used to study the chemical freezeout dynamics within the framework of a statistical model.
\end{abstract}

\section{Introduction}

To understand the properties of matter under extreme conditions of high temperature or density, heavy-ion collision experiments are conducted at RHIC in BNL and the LHC in CERN. These are the conditions, in which the deconfined phase of QCD matter, the Quark-Gluon Plasma (QGP), is created. It is conjectured that the formed hot and dense partonic matter rapidly expands and cools down. During the evolution it undergoes a transition back to the hadronic matter $[1,2]$. Both RHIC and LHC have confirmed the formation of the QGP in central $\mathrm{Au}+\mathrm{Au}$ and $\mathrm{Pb}+\mathrm{Pb}$ collisions [3, 4]. In $\mathrm{QCD}$, there are three conserved charges, baryon number $B$, electric charge $Q$ and strangeness $S$. Thus the equilibrium thermodynamic state of QCD matter is completely determined by temperature $T_{c h}$ and the three chemical potentials $\mu_{B}, \mu_{Q}$, and $\mu_{S}$ corresponding to $B, Q$ and $S$ respectively. The QCD phase diagram is plotted with the temperature $(T)$ as a function of baryon chemical potential $\left(\mu_{B}\right)$ [5]. From finite-temperature QCD calculations on the lattice it is theoretically established that the transition from QGP to a hadron gas happens at high temperature and $\mu_{B}$ close to zero and is a cross-over [6]. Several QCD-based calculations [7] suggest existence of first-order phase transition at a lower $T$ and large $\mu_{B}$. Therefore, there should be an end point for the first-oder phase transition in the QCD phase diagram, known as the critical point. Several QCD based models and also calculations on lattice predict the existence of the critical point at high $\mu_{B}$ [8] and its exact location depends on the different model assumptions [9, 11-13]. It is worth to mention that not all QCD-based models or lattice groups do predict the existence of critical point [14].

\footnotetext{
ae-mail: sabita@rcf.rhic.bnl.gov
}

Theoretically, the phase diagram is explored through nonperturbative QCD calculations on lattice which indicates the energy scale can be explored experimentally. Now to explore various aspects of the QCD phase diagram[15] such as the search for the signals of phase boundary, and the search the location of the critical point has become one of the main goals of the BES program at RHIC. The RHIC BES program started a test run of $\mathrm{Au}+\mathrm{Au}$ collisions at $\sqrt{s_{N N}}=9.2 \mathrm{GeV}$ [22]. This was followed by setting a number of observables for the physics goals [23]. The first phase of the BES program started in the year in the year 2010 and 2011 in $\mathrm{Au}+\mathrm{Au}$ collisions at $\sqrt{s_{N N}}=7.7,11.5$, 19.6, 27, and $39 \mathrm{GeV}$. In addition, the NA49 collaboration has reported evidence that the onset of de-confinement occurs close to $\sqrt{s_{N N}}=7.7 \mathrm{GeV}$ [24]. The process of hadron decoupling from an interacting system in heavyion collisions is known as freeze-out. They are of two types, kinetic and chemical freeze-out. We will present here the study on chemical freeze-out, characterised by temperature $\left(\mathrm{T}_{c h}\right)$ and baryon chemical potential $\left(\mu_{B}\right)$, when inelastic collisions cease and the particle yields become fixed. The $T_{c h}$ and $\mu_{B}$ can be extracted using the particle ratios obtained from the measured particle yields and comparing with model calculations which assume the system is in chemical and thermal equilibrium.

Here we will discuss the identified particle production produced in $\mathrm{Au}+\mathrm{Au}$ collisions at BES center-of-mass energies $\sqrt{s_{N N}}=7.7,11.5,19.6,27$, and $39 \mathrm{GeV}[25,26]$. The energy and centrality dependence of particle yields, ratios of particle yields, and average transverse mass will also be discussed for the above energies. The mid-rapidity yields of charged pions $\left(\pi^{ \pm}\right)$, charged kaons $\left(K^{ \pm}\right)$, protons $(p, \bar{p}), K_{S}^{0}$, Lambdas $(\Lambda, \bar{\Lambda})$ and Cascades $\left(\Xi^{-}, \bar{\Xi}^{+}\right)$have been used for the chemical freeze-out 

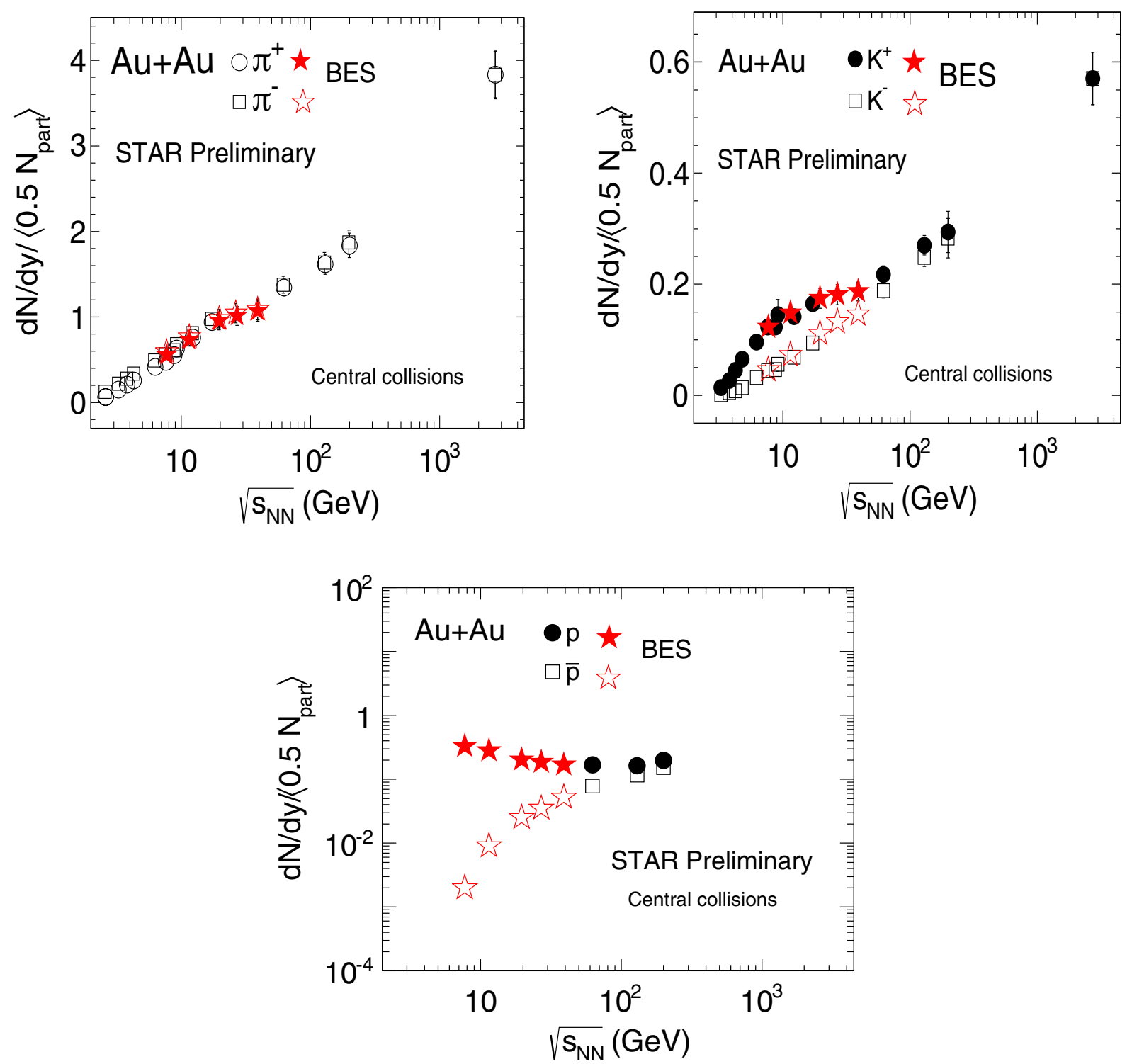

Figure 1. $d N / d y$ of $\pi^{ \pm}, K^{ \pm}, p$, and $\bar{p}$ scaled by $\left\langle 0.5 N_{\text {part }}\right\rangle$ as a function of center of mass energy $\left(\sqrt{s_{N N}}\right)$ including collisions at BES energies (red points) along with AGS [16-18], SPS [19], top RHIC [20], and LHC energies [21] for central collisions. Errors are statistical and systematic errors added in quadrature.

study [25-27]. An equilibrium thermal model, such as THERMUS [28] has been quite successful at reproducing the particle production in heavy-ion collisions [29-31]. To extract the chemical freeze-out temperature $\left(T_{c h}\right)$, baryon chemical potential $\left(\mu_{B}\right)$, strangeness chemical potential $\left(\mu_{S}\right)$ and strangeness saturation factor $\left(\gamma_{S}\right)$, the experimental particle ratios are used in both grand canonical ensemble (GCE) and strangeness canonical ensemble (SCE) approach of the model. The centrality and energy dependence of $T_{c h}, \mu_{B}, \mu_{S}, \gamma_{S}$ in $\mathrm{Au}+\mathrm{Au}$ collisions at the above BES energies are studied.

\section{Results}

\subsection{Particle Yields}

The mid-rapidity $(|y|<0.1)$ BES data presented here are obtained using the STAR Time Projection chamber (TPC) and Time-Of-Flight (TOF) detectors [32]. The particles are identified by measuring the specific ionisation energy loss in the TPC and the particle velocities using TOF as a function of momentum. Figure 1 show the $d N / d y$ normalised to the average number of participant nucleus $\left(d N / d y /\left\langle 0.5 N_{\text {part }}\right\rangle\right)$ vs. $\sqrt{s_{N N}}$ for $\pi^{ \pm}, K^{ \pm}, p$, and $\bar{p}$ for 0 $5 \%$ centrality at BES energies and those from published data at AGS [16-18], SPS [19], RHIC [20], and LHC energies [21]. The errors on the points include both statistical 


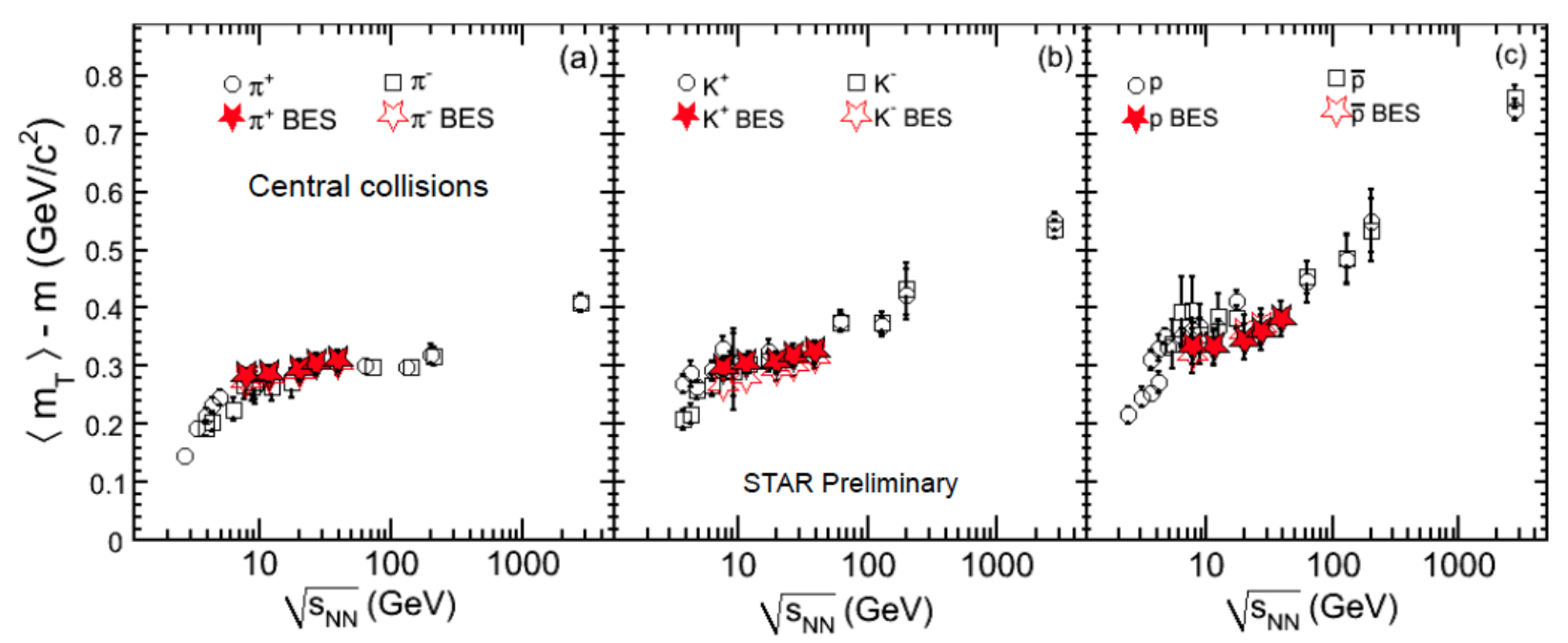

Figure 2. The variation of $\left\langle m_{T}\right\rangle-m$ of charged pions, kaons, and (anti)protons vs. $\sqrt{s_{N N}}$ measured in $|y|<0.1$ in collisions at BES energies along with AGS [16-18], SPS [19], RHIC [20], and LHC energies [21] for central collisions. Errors are statistical and systematic errors added in quadrature.

and systematic errors. The mid-rapidity yields of charged pions, kaons, and anti-protons increases with increasing collision energy whereas the protons yields decreases with increase of collision energy. The results from BES data are in agreement with the general energy dependence trend observed at AGS, SPS, top RHIC, and LHC energies.

\subsection{Average Transverse Mass}

Figure 2 shows the energy dependence of $\left\langle m_{T}\right\rangle-m$ for $\pi^{ \pm}, K^{ \pm}, p$, and $\bar{p}$ in central $(0-5 \%) \mathrm{Au}+\mathrm{Au}$ collisions at BES energies along with data for $\mathrm{Pb}+\mathrm{Pb} / \mathrm{Au}+\mathrm{Au}$ collisions from AGS [16-18], SPS [19], RHIC [20], and LHC energies [21].

The $\left\langle m_{T}\right\rangle-m$ increases with energy at lower energies, remains almost constant at SPS and lower RHIC BES energies and then increases again towards higher energies up to LHC energy. The behaviour of $\left\langle m_{T}\right\rangle-m$ might indicate the onset of the phase transition [33]. If the system is assumed to be thermodynamic, $\left\langle m_{T}\right\rangle-m$ and $\sqrt{s_{N N}}$ can be related to temperature and entropy of the system $\left(d N / d y \propto \log \sqrt{s_{N N}}\right)$, respectively. Based on this, the constant value of $\left\langle m_{T}\right\rangle-m$ can be interpreted as a signature of first order phase transition. $\left\langle m_{T}\right\rangle-m$ could be sensitive to several other effects [34]. It is observed that with increasing centrality the values of $\left\langle m_{T}\right\rangle$ increase, indicating that the development of stronger collective motion in more central collisions and the difference between particles also increases.

\subsection{Particle Ratios}

Figure 3 shows the variation of different particle ratios as a function of center-of-mass energy in $\mathrm{Au}+\mathrm{Au}$ collisions at BES energies and its comparison with the results from AGS [16-18], SPS [19], RHIC [20], and LHC energies [21]. The variation of $K^{-} / K^{+}$as a function of $\bar{p} / p$ for $0-5 \%$ centrality from SPS-LHC energies has been shown. Errors are statistical and systematic errors added in quadrature.

As the collision energy increases, $\pi^{-} / \pi^{+}$ratio decreases to unity whereas $K^{-} / K^{+}$ratio rise systematically. At higher energy, pair production, which results in the same number of positive and negative pions dominates the resonance decays. Following this logic, the $\pi^{-} / \pi^{+}$ratio is supposed to reach unity as the energy goes up. The $K^{-} / K^{+}$ratio is indicative of the relative contribution of associated and pair production. The associated production mechanism can only produce $K^{+}$via $N+N \rightarrow N+X+K^{+}$, $\pi+N \rightarrow X+K^{+}$where $N$ is nucleon and $X$ is hyperon ( $\Lambda$ or $\Xi)$, while the pair production mechanism produces $K^{+}$and $K^{-}$via $N+N \rightarrow N+N+K^{+}+K^{-}$. The rise of $K^{-} / K^{+}$ ratio as a function of energy can be attributed to the nature of kaon production channels. At lower energy the associated production dominates, due to a lower energy threshold. As the energy increases, the pair production which produces the same number of $K^{+}$and $K^{-}$becomes more significant. With increasing energy, the net baryon density decreases and thus the associated production of $K^{+}$also decreases, while pair production increases due to gluongluon fusion into strange quark-antiquark pairs [35, 36]. All these results combined, when compared with previous experiments, seem to be consistent with an enhancement in the strangeness production. At lower energies due to the non-zero net baryon density in the collisions zone, the associated production of kaons with hyperons will be different from these produced with anti-hyperons.

The $K^{-} / K^{+}$, which represents net-strange chemical potential $\left(\mu_{S}\right)$ vs. $\bar{p} / p$, representing net-baryon chemical potential $\left(\mu_{B}\right)$ for $0-5 \%$ centrality in $\mathrm{Au}+\mathrm{Au}$ collisions together with results from top RHIC, has been shown in Fig. 3. Both ratios are affected by the net baryon con- 

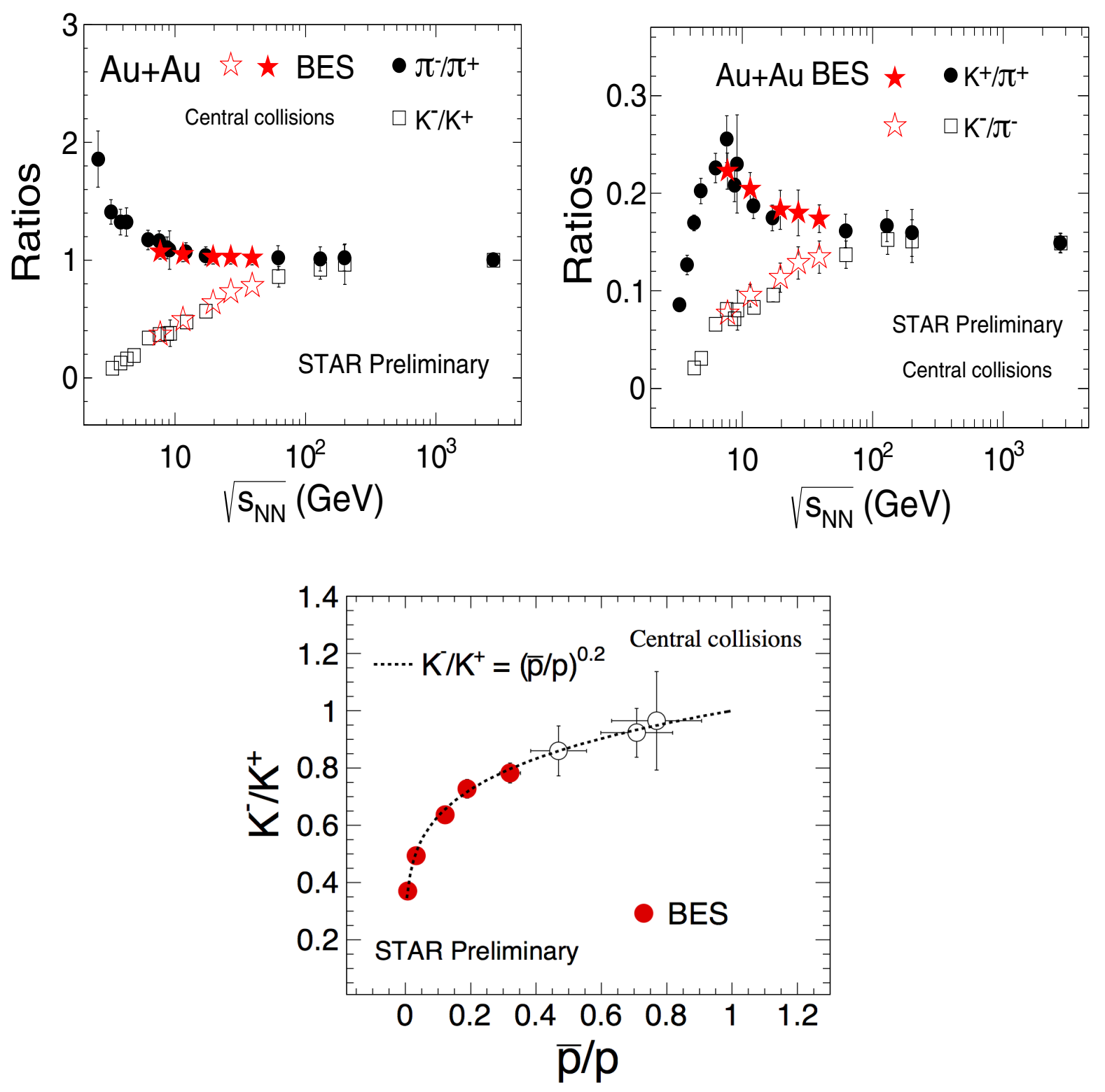

Figure 3. Particle ratios as a function of center of mass energy in $\mathrm{Au}+\mathrm{Au}$ collisions at BES energies. The results are compared with AGS [16-18], SPS [19], RHIC [20], and LHC energies [21]. The variation of $K^{-} / K^{+}$as a function of $\bar{p} / p$ for $0-5 \%$ centrality from SPS-LHC energies. Errors are statistical and systematic errors added in quadrature.

tent; they show a strong correlation. In a hadron gas, both chemical potentials, $\mu_{S}$ and $\mu_{B}$ depends on temperature and they follow the relation $\mu_{S}=\mu_{B} / 3$ [37]. It is worth noting that at low energies, the absorption of antiprotons in the baryon-rich environment plays a vital role.

\subsection{Chemical freeze-out}

At chemical freeze-out, inelastic collisions among the particles stop, particle yields and ratios of particle yields get fixed. Particle ratios are calculated taking the ratios from the measured integrated yields. A set of different particle ratios which involves the particle yields of $\pi^{ \pm}, K^{ \pm}, p$, $\bar{p}, \Lambda, \bar{\Lambda}, \Xi^{-}, \bar{\Xi}^{+}$can be collectively used to extract the information on the chemical freeze-out conditions. The extraction of freeze-out parameters is very senstive to the contribution of weak decays, commonly called feed-down. Experimentally different particles are corrected in different ways. At STAR, proton yields have not been corrected for feed-down contributions, and are commonly called "inclusive", where as $\pi$ and $\Lambda$ yields have been corrected for the feed-down from $K_{S}^{0}, \Xi$ and $\Xi^{0}$ weak decays, respectively. In model, the appropriate feed-down as in experimental data has been considered. Different freezeout parameters are extracted using those ratios comparing with the corresponding ratios calculated in the THERMUS model assuming chemical equilibrium. In the THERMUS model, in thermodynamical equilibrium, the particle abun- 

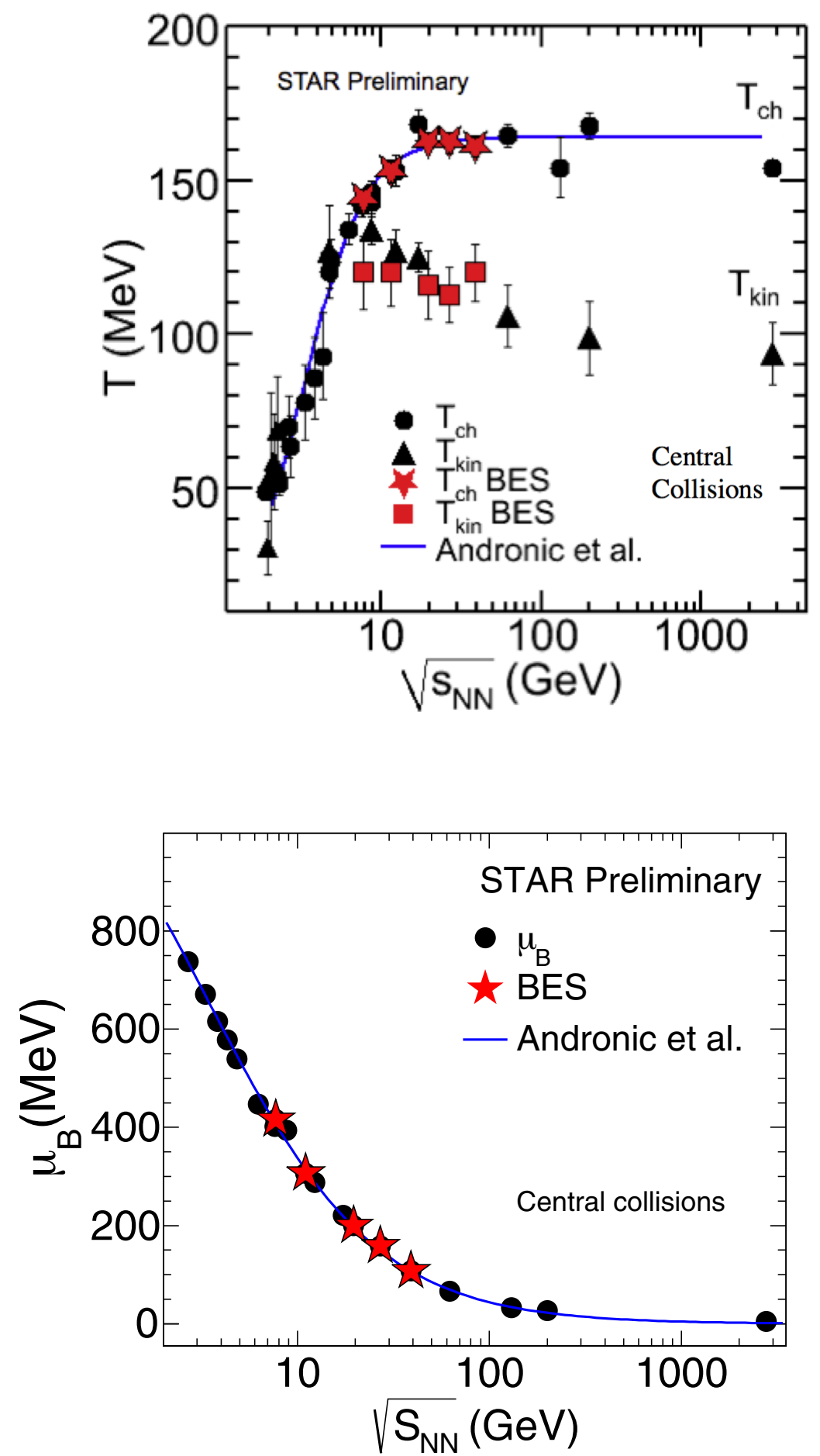

Figure 4. The energy dependence of the extracted parameters $T_{c h}$ (upper) and $\mu_{B}$ (lower) for central collisions. The curves represent the theoretical predictions [38].

dance of $i$-th particle $\left(N_{i}\right)$ in a system of volume $V$ can be given by [28]

$$
\frac{N_{i}}{V}=\frac{T m_{i}^{2} g_{i}}{2 \pi^{2}} \sum_{k=1}^{\infty} \frac{( \pm 1)^{k+1}}{k} \exp \left(\beta k \mu_{i}\right) K_{2}\left(\frac{k m_{i}}{T}\right),
$$

where $T$ is the chemical freeze-out temperature, $m_{i}$ is the particle mass, $g_{i}$ is the degeneracy, $\beta \equiv \frac{1}{T}, K_{2}$ is the second order Bessel function and $\mu_{i}$ is the chemical potential of hadron species $i$ which is given by $\mu_{i}=B_{i} \mu_{B}+S_{i} \mu_{S}+Q_{i} \mu_{Q}$

where $B_{i}, S_{i}$ and $Q_{i}$ are the baryon number, strangeness and charge, respectively, of species $i$ and $\mu_{B}, \mu_{S}$ and $\mu_{Q}$ are the corresponding chemical potentials. In the model calculations of particle ratios show a very good agreement with data at BES energies studied for all centralities [25]. 
The upper plot in Fig. 4 shows that the chemical freezeout temperature $T_{c h}$ for central heavy-ion collisions as a function of energy and it increases with the increase of energy. The lower plot of Fig. 4 shows the decrease of $\mu_{B}$ with increasing collision energy. These measurements in the BES program have covered a wide range at RHIC, from around $\mu_{B}=20 \mathrm{MeV}$ to about $420 \mathrm{MeV}$ in the QCD phase diagram. The large value of $\mu_{B}$ at midrapidity may indicate the formation of a high net-baryon density matter, which is expected to reach a maximum value around $7.7 \mathrm{GeV}$ [39]. The temperature of kinetic freeze-out $\left(T_{k i n}\right)$, where elastic collisions stop and particle spectra get fixed, obtained using a Blast-Wave fit to the identified transverse momentum spectra is found to takes place after chemical freeze-out [40].

\subsection{Summary}

The identified particle production have been discussed in central $\mathrm{Au}+\mathrm{Au}$ collisions at all BES energies $\left(\sqrt{s_{N N}}=\right.$ $7.7,11.5,19.6,27,39 \mathrm{GeV})$. The energy dependence of identified particle yields and ratios, the average transverse mass are discussed briefly. The measured particle ratios at mid-rapidity have been used to extract the parameters of chemical freeze-out, where the inelastic collisions of hadrons have stopped. The chemical freeze-out parameters reflect the properties of the system at earlier stage compared with that at kinetic freeze-out.

The BES energies along with the top RHIC energies have allowed one to access the region of the QCD phase diagram covering a wide range of baryon chemical potential $\left(\mu_{B}\right)$ from 20 to $420 \mathrm{MeV}$ corresponding to $\mathrm{Au}+\mathrm{Au}$ collision energies from $\sqrt{s_{N N}}=200$ down to $7.7 \mathrm{GeV}$, respectively. An important $T_{c h}, \mu_{B}$ point will be added at $\sqrt{s_{N N}}=14.5 \mathrm{GeV}$ as a part of BES-I data which was taken in 2014 to cover a large gap of $\mu_{B}$ between 11.5 and 19.6 GeV in phase diagram. Current lattice QCD calculations suggest that key features of the phase diagram like the critical point and the first-order phase transition may lie within the $\mu_{B}$ reach of the RHIC BES program [41]. In BES phase-II, a systematic measurement of the yields of a variety of produced hadrons versus rapidity, centrality, and beam energy will address various questions about the evolution of the hadron yields between the initial hadronization and the final thermal equilibrium [42] and about the possibility of successive hadronization [43]. This could lead to further understanding and refinement of the statistical models. In addition to BES program at RHIC [44], new experimental facilities have been designed at the Facility for Antiproton and Ion Research (FAIR) at GSI and Nuclotron-based Ion Collider fAcility (NICA) at JINR in order to search for the QCD critical point [45].

\section{References}

[1] B. B. Back et al., Nucl. Phys. A 757, 28 (2005); I. Arsene et al. (BRAHMS Collaboration), Nucl. Phys. A 757, 1 (2005); J. Adams et al. (STAR Collaboration), Nucl. Phys. A 757, 102 (2005).
[2] K. Adcox et al. (PHENIX Collaboration), Nucl. Phys. A 757, 184 (2005) M. Gyulassy and L. McLerran, Nucl. Phys. A 750, 30 (2005).

[3] S. Chatrchyan et al., JHEP 05, 063 (2012); B. Abelev et al., Phys. Rev. Lett. 109, 072301 (2012).

[4] R. Singh et al., Advances in High Energy Physics 2013 761474) (2013).

[5] X Luo, B Mohanty, HG Ritter, N Xu. Science 332 (6037), 1525-1528, (2011).

[6] Y. Aoki et al., Nature 443, 675 (2006).

[7] S. Ejiri, Phys. Rev. D 78074507 (2008).

[8] K. Rajagopal and F. Wilczek, arXiv:hepph/0011333.

[9] M. Stephanov, Prog. Theor. Phys. Suppl. 153, 139 (2004); Int. J. Mod. Phys. A 20, 4387 (2005);

[10] E. S. Bowman et al., Phys. Rev. C 79 015202(2009).

[11] Z. Fodor et al., JHEP 50, 0404 (2004).

[12] R. V. Gavai and S. Gupta, Phys. Rev. D 78, 114503 (2008); Phys. Rev. D 78, 14503 (2008); Phys. Rev. D 71, 114014 (2005).

[13] Anyi Li et al., arXiv:0908.1155.

[14] G. Endrodi et al., J. High Energy Phys., vol. 1104, p. 1, 2011. 75; S. Borsanyi, arXiv:1210.6901v1.

[15] P. Braun-Munzinger et al., Rev. Mod. Phys. 81, 1031 (2009); B. Mohanty, New J.Phys., 13, 065031 (2011).

[16] L. Ahle et al. (E866 Collaboration and E917 Collaboration), Phys. Lett. B 490, 53 (2000); L. Ahle et al. (E866 Collaboration and E917 Collaboration), Phys. Lett. B 476, 1 (2000).

[17] J. L. Klay et al. (E895 Collaboration), Phys. Rev. Lett. 88, 102301 (2002); J. Barrette et al. (E877 Collaboration), Phys. Rev. C 62, 024901 (2000);

[18] L. Ahle et al. (E802 Collaboration), Phys. Rev. C 60, 064901 (1999); L. Ahle et al. (E802 Collaboration and E866 Collaboration), Phys. Rev. C 60, 044904 (1999); L. Ahleet al. (E802 Collaboration), Phys. Rev. C 57, 466 (1998).

[19] S. V. Afanasiev et al. (NA49 Collaboration), Phys. Rev. C 66, 054902 (2002); C. Alt et al. (NA49 Collaboration), Phys. Rev. C 77, 024903 (2008); ibid. 73, 044910 (2006); T. Anticic et al. (NA49 Collaboration), Phys. Rev. C 69, 024902 (2004).

[20] J. Adams et al. (STAR Collaboration), Phys. Rev. Lett. 91, 072304 (2003); Phys. Rev. Lett. 91, 172302 (2003); B. I. Abelev et al. (STAR Collaboration), Phys. Rev. Lett. 97, 152301 (2006); Phys. Lett. B 655, 104 (2007), J. Adams et al. (STAR Collaboration), Phys. Rev. Lett. 92, 112301 (2004); B. I. Abelev et al. (STAR Collaboration), Phys. Rev. C 79, 034909 (2009).

[21] B. Abelev et al. (ALICE Collaboration), Phys. Rev. Lett 109, 252301 (2012); B. Abelev et al. (ALICE Collaboration), Phys. Rev. C 88, 4, 044910 (2013).

[22] B. I. Abelev et al. (STAR Collaboration), Phys. Rev. C 81, 024911 (2010). 
[23] B. I. Abelev et al., (STAR Collaboration) STAR Internal Note, SN0493 (2009).

[24] C. Alt et al. (NA49 collaboration), Phys. Rev. C 77, 024903 (2008).

[25] S. Das (STAR Collaboration), Nucl. Phys. A, 904905, 891C, (2013); J. Phys.: Conf. Ser. 509, 012066 (2014).

[26] L. Kumar (STAR Collaboration) ,J. Phys. G Nucl. Part. Phys., 38124145 (2011).

[27] X. Zhu (STAR Collaboration) Acta Phys. Polon. B, Proc. Supp. 5, 213-218 (2012).

[28] S. Wheaton and J. Cleymans, Comput. Phys. Commun. 180, 84 (2009).

[29] P. Braun-Munzinger et al. Physics Letters B, vol. 365, 1-6 (1996); G. D. Yen and M. I. Gorenstein, Physical Review C, vol. 59, 12 article 2788 (1999).

[30] F. Becattini et al. Physical Review C, vol. 64,Article ID024901 (2001); A. Andronic et al., Nuclear Physics A, vol. 772, no. 3-4, pp. 167-199 (2006);

[31] F. Becattini, et al., TheEuropean Physical Journal C, vol. 66, no. 3-4, pp. 377-386 (2010).

[32] M. Anderson et al., Nucl. Instr. Meth. A 499, 659 (2003); W. J. Llope et al., Nucl. Instr. Meth. B 241, 306(2005)
[33] M. I. Gorenstein, M. Gazdzicki and K. A. Bugaev, Phys. Lett. B 567, 175 (2003).

[34] B. Mohanty et al., Phys. Rev. C 68, 021901 (2003).

[35] J. Rafelski, and B. Muller, Phys. Rev. Lett. 481066 (1982).

[36] R. Koch et al., Phys. Rep. 142167 (1986).

[37] I. G. Bearden et al. (BRAHMS Collaboration), Phys. Rev. Lett. 90, 102301 (2003).

[38] A. Andronic, P. Braun-Munzinger and J. Stachel, Nucl. Phys. A 834, 237C (2010).

[39] J. Randrup and J. Cleymans, Phys. Rev. C 74, 047901 (2006).

[40] L. Kumar (for the STAR Collaboration); arXiv:1408.4209

[41] K. Fukushima and T. Hatsuda, Rept. Prog. Phys. 74, 014001 (2011); M. Alford, PoS (LAT2006) 001. .

[42] F.Becattini, et. al, Phys. Rev. Lett. 111, 082302 (2013).

[43] S. Chatterjee, et. al, Phys. Lett. B 727, 554 (2013).

[44] M. M. Aggarwal et al. (STAR Collaboration), Phys. Rev. Lett. 105, 022302(2010); L. Adamczyk et al. (STAR Collaboration), Phys. Rev. Lett. 112, 032302 (2014).

[45] Johann M. Heuser for CBM Collaboration, Nucl.Phys.A 830, 563c-566c, (2009). 